\title{
El papel de las ciencias exactas en la formación de restauradores en Colombia
} Mario Omar Fernández R. *

David Cohen D.**

\section{Resumen}

El presente artículo corresponde a una reflexión acerca de la formación profesional de restauradores en Colombia, destacando el aporte de las ciencias naturales dentro del proceso y su contribución en el establecimiento de un esquema de trabajo interdisciplinario entre científicos y conservadores. Expone la labor llevada a cabo por el Laboratorio de Ciencias Naturales desde su creación en 1997 en el estudio de obras de patrimonio cultural y su relación académica con el pregrado, así como el impulso que ha brindado al desarrollo de otras áreas y profesionales de la conservación.

Otro de los temas tratados corresponde a la evolución de la conservación preventiva dentro del programa de estudios y las implicaciones que ha tenido la apertura y consolidación de esta cátedra como un área del conocimiento, fundamental en la formación de conservadores - restauradores.

Se presentan algunos casos de estudio y experiencias en conservación preventiva de museos e iglesias de diferentes regiones del país, como ejemplos de proyectos exitosos de gran impacto social, ejecutados con bajo presupuesto y en poco tiempo.

Palabras clave: Ciencias exactas. Restauración. Conservación preventiva.

The role of natural sciences in the professional training of restorers in Colombia

\begin{abstract}
The following paper presents a brief reflection about the professional formation of restorers in Colombia, highlighting the contribution of the natural sciences within the process, introducing an interdisciplinary frame of work. The article shows the work made by the Laboratory of Natural Sciences since its creation on 1997, studying Cultural Heritage objects on a close relationship with the academic training of
\end{abstract}


restorers, and involving other professionals related to the Heritage's conservation. Another topic corresponds to the evolution of the Preventive Conservation chair and the implications it has had into the formation of conservators - restorers. Some cases are presented, as well as successful preventive conservation experiences on museums and churches, located on different places of the country.

\section{Key - words: Natural sciences. Restoration. Preventive conservation}

\section{Introducción}

A lo largo del proceso de formación de restauradores en Colombia que ya cumple treinta años, las ciencias naturales han jugado un papel preponderante participando de manera activa en diversas áreas relacionadas con la conservación del patrimonio cultural.

El trabajo de los científicos ha permitido generar estrategias pedagógicas enfocadas a promover un marco en donde diversas disciplinas convergen desde su propia mirada, brindando aportes para entender la compleja problemática que implica el estudio del patrimonio y la proposición de soluciones de conservación - restauración ajustadas a los condicionamientos que impone un contexto como el de Colombia, en el que la inversión por parte del Estado en la recuperación de los bienes culturales no es una prioridad.

Parte de la labor de los científicos se ha centrado en el Laboratorio de Ciencias Naturales de la Universidad Externado de Colombia que en los últimos años, se ha consolidado como un espacio especializado en el estudio de los bienes culturales que no sólo se limita a la docencia, sino que articula diversos profesionales que interactúan con el patrimonio, abriendo así nuevas perspectivas interdisciplinarias de trabajo.

El Laboratorio de ciencias también ha jugado un papel importante en la conservación preventiva, generando proyectos integrales que involucran la evaluación de riesgos, el diagnóstico de las colecciones, el mejoramiento de las condiciones ambientales y 
posteriormente la restauración de obras dentro de los talleres del pregrado. Estos trabajos han fortalecido la cátedra de conservación preventiva que fue creada en el año 1997, coincidiendo con la creación del Consorcio Latinoamericano de Conservación Preventiva iniciativa del Instituto de Conservación Getty.

\section{Breve historia de la profesión}

Para entender como ha sido el desenvolvimiento de la conservación del patrimonio cultural y en particular de la disciplina de la restauración en Colombia, es necesario comprender, cómo ha sido la formación profesional y cuál ha sido la participación de las ciencias naturales en el proceso.

En la década de los setenta, comienza la formación de restauradores con la aparición de COLCULTURA (1) y el nacimiento del Centro Nacional de Restauración. A partir de los años ochenta, la Escuela ajusta su programa académico reestructurando el plan de estudios a cinco años y consolidando los talleres de restauración existentes: pintura de caballete, madera y escultura policromada, pintura mural, textiles, cerámica y bienes gráficos y documentales. "Ésta etapa inicial se caracterizó por el paso de un trabajo empírico artesanal, que conllevaba una técnica primaria, a uno técnico operativo con apoyo científico, que buscaba lograr mayor seguridad y precisión en las intervenciones." (CADAVID, 1994, p. 33).

En 1992, buscando fortalecer el esquema de formación, se suscribe un convenio con la Universidad Externado de Colombia para ofrecer, el programa de restauración como pregrado profesional, entrando en plena vigencia en 1994 con el ingreso de la primera promoción de estudiantes. En sus inicios, el programa conjunto entre ambas entidades desarrolló el proceso de formación dentro de la academia, a excepción de los talleres que continuaron impartiéndose en las instalaciones del Centro hasta 1997, cuando se estructura y se pone en marcha, el primer taller de restauración dentro de la Universidad.

Desde ese momento, los talleres han seguido creciendo y gracias al trabajo conjunto entre científicos y restauradores, fue posible en 2001 con el apoyo de la ECRYM (2) crear el taller de restauración de metales inexistente hasta esa fecha en Colombia y 
concebido para dar respuesta a las necesidades del patrimonio mueble del país, que involucra una amplia tradición orfebre y metalúrgica.

Desde que comenzó el programa en la Universidad Externado hasta la fecha, se han formado ciento noventa profesionales y se han analizado, diagnosticado e intervenido dentro de los talleres de restauración, setecientas setenta obras del patrimonio cultural mueble colombiano. Estos bienes, pertenecen a entidades del sector público o bien, a entidades que siendo particulares, permiten el acceso del público a sus colecciones; enfoque que ha permitido a la Universidad, establecer importantes vínculos interinstitucionales dentro del campo cultural generando un gran impacto a nivel nacional y beneficiando a museos, casas de cultura, archivos e iglesias.

Cada una de las obras que hacen parte de los casos de estudio de los talleres, es abordada por un equipo docente compuesto por diversos profesionales, de los cuales los científicos contribuyen en gran medida en el proceso de diagnóstico que llevan a cabo los estudiantes como paso previo a la presentación de sus propuestas de restauración, colaborando en el entendimiento de las técnicas, materiales y procesos de alteración que sufren los bienes culturales. Este diálogo así como las necesidades derivadas de la evolución misma del proceso de formación, condujo finalmente a la creación de los "módulos científicos" al interior de cada uno de los talleres aportando conceptos y metodologías científicas necesarios para el entendimiento de la materialidad de cada uno de los soportes y sus procesos de alteración. Los módulos han servido como espacio de conocimiento y discusión de las problemáticas específicas para los diferentes tipos de objetos.

Así mismo, el Laboratorio asesora muchas de las intervenciones que se llevan a cabo en los talleres, respondiendo preguntas acerca de los tratamientos que se realizan y desarrollando investigaciones propias en esta área. (3)

\section{El Laboratorio de Ciencias Naturales}

El Laboratorio de Ciencias de la Facultad de Restauración fue diseñado y creado para apoyar las actividades académicas del programa de Conservación y Restauración de Bienes Muebles. Desde el año 1998 el Laboratorio asumió la 
realización del estudio de las obras que se intervienen en los talleres de restauración, trabajos de investigación, y con posterioridad comenzó a realizar servicios científico-técnicos a proyectos del Ministerio de Cultura y al público en general; se han estudiado más de 1.000 bienes culturales de Colombia y de otros países como Bélgica (4), Bolivia (5), Chile (6), México (7) y Panamá (8) entre otros.

Asumir nuevos compromisos con una infraestructura adquirida fundamentalmente para la práctica docente, ha sido un reto. Por tal motivo y ante la posibilidad de utilizar la infraestructura del laboratorio del antiguo Centro Nacional de Restauración, el Laboratorio realizó una readecuación de tres espacios del edificio de la facultad para albergar los equipos y ponerlos en función del programa académico y del estudio del patrimonio cultural colombiano.

El Laboratorio de Ciencias de la Facultad de Restauración cuenta con varias áreas de trabajo. La primera de ellas es el área de microscopía y microquímica, técnicas analíticas que han permitido con pocos recursos estudiar un número elevado de bienes culturales. Así mismo se han desarrollado nuevas técnicas de análisis en función de las necesidades que se han ido generando. Los análisis que más se realizan en esta área van dirigidos a la identificación de fibras textiles y papeleras, la identificación de pigmentos, y la composición de preparaciones y morteros de construcción.

El área de petrografía ha contado con el apoyo de los geólogos cuyos aportes han sido muy valiosos. Con el estudio de secciones delgadas de material pétreo, cerámico y de morteros y argamasas, se han realizado importantes contribuciones a proyectos de investigación y restauración de significativos monumentos arquitectónicos, colecciones arqueológicas y parques arqueológicos.

El área de metalografía de reciente creación en el Laboratorio, ha implementado las técnicas metalográficas que han sido de gran utilidad en el estudio de monumentos en espacio público, artes decorativas y objetos arqueológicos de naturaleza metálica. Los estudios se dirigen fundamentalmente a la identificación de la composición de metales y aleaciones, técnicas de elaboración y productos de corrosión. 
El trabajo de los biólogos ha sido fundamental para el estudio de los procesos de biodeterioro y el establecimiento de programas de control de plagas. Ésta área de microbiología, presenta una gran demanda en Colombia debido a las diferentes regiones geográficas del país, cada una con variables muy disímiles y situaciones complejas.

El Laboratorio de ciencias se ha convertido en un espacio de convergencia de diferentes disciplinas que se ocupan del estudio y la conservación de los bienes culturales. En la medida que posee un amplio portafolio de servicios y experiencia en el estudio del patrimonio, diversos públicos se benefician de las investigaciones y servicios que presta; los conservadores - restauradores de bienes muebles por supuesto ocupan un espacio sobresaliente en estas dinámicas interdisciplinarias, pero otros profesionales también interactúan con los científicos; arquitectos restauradores, museólogos, historiadores, arqueólogos y coleccionistas privados acuden con frecuencia planteando preguntas a las ciencias exactas.

Quizás como uno de los logros más destacables es el haber implementado un sistema de documentación que se convierte en una valiosa fuente de información para los investigadores de diversas disciplinas. La conservación y ordenamiento de las muestras analizadas también se constituyen en un importante recurso documental para investigaciones en el campo del patrimonio cultural. (9)

\section{La conservación preventiva}

La cátedra de conservación preventiva se inicia en el programa de Conservación y Restauración de Bienes Muebles en el año 1997 y desde entonces ha tenido un acelerado desarrollo y un importante impacto en la conservación del patrimonio mueble colombiano.

Denominamos conservación preventiva al conjunto de acciones dirigidas a disminuir los riesgos de deterioro de las colecciones convirtiéndose en los últimos años, en una posición ética de los profesionales que preservan el patrimonio cultural del país. 
La asignatura ha tenido una estrecha relación con la problemática del patrimonio que se alberga en museos, archivos, bibliotecas e instituciones religiosas, lo que ha permitido preparar a los estudiantes para enfrentar las crecientes necesidades de conservación de los bienes muebles en el contexto colombiano y latinoamericano.

Vale la pena resaltar la calidad de los trabajos de investigación, algunos han permitido resolver problemas puntuales de colecciones en importantes instituciones culturales y religiosas, otros ya han sido publicados. (10)

La cátedra de conservación preventiva desde su creación ha tenido estrechos vínculos con entidades nacionales como la Red Nacional de Museos, de la cual ha recibido importante apoyo. Se destaca en el orden internacional la participación en el Consorcio Latinoamericano de Formación en Conservación Preventiva cuya meta principal es mejorar la conservación preventiva en Latinoamérica a través del establecimiento de una red de especialistas reforzando las capacidades existentes de las instituciones y los individuos que son miembros y que se encuentran involucrados en actividades de capacitación. El Consorcio estuvo guiado inicialmente por el Instituto de Conservación Getty de EEUU, con la participación de Chile, Cuba, México, Argentina y Brasil. (11)

Las prácticas académicas se han constituido en motor de crecimiento de la asignatura y a su vez han incidido satisfactoriamente en la conservación de diferentes grupos de objetos patrimoniales.

Los primeros trabajos se dirigieron a probar herramientas metodológicas para la evaluación de riesgos de deterioro (12) y a la familiarización con la situación real de los museos e iglesias colombianos, generando propuestas puntuales a nivel de exhibición, depósito, y planes de emergencia, que sirvieron a las instituciones para mejorar las condiciones de conservación de sus colecciones.

Estos trabajos sirvieron de base para la formulación de proyectos semestrales en una sola institución atados al desarrollo académico de la asignatura. Aunar todos los esfuerzos de estudiantes y profesores en un solo museo o iglesia, permitió la 
profundización en todos los temas dando la posibilidad de realizar prácticas académicas de gran impacto y acogida por parte de los funcionarios y el público.

Este esquema pedagógico ha permitido, además de fortalecer el conocimiento teórico-práctico de los restauradores, mejorar significativamente las condiciones de preservación de varias colecciones en diferentes ciudades del país.

Se realizaron trabajos de conservación preventiva en la iglesia de Nuestra Señora de Egipto y en la iglesia Museo Santa Clara, de la ciudad de Bogotá, también fueron objeto de estudio: el Museo arqueológico de Sogamoso, el Museo del Carmen de Villa de Leyva y el Museo de Arte Religioso Julio Franco Arango de Duitama; el Museo arquidiocesano de Pamplona, en el Norte de Santander; el Museo de Arte del Tolima (MAT) en Ibagué y los Museos Histórico y de Arte Contemporáneo de la Quinta de San Pedro Alejandrino en Santa Marta.

En todas estas instituciones se evaluaron aspectos administrativos y de gestión para apreciar su incidencia en la conservación de las colecciones. Se revisaron los planes de emergencia y la existencia de inventarios y registros. Se prestó especial atención al estudio del clima, se realizaron mediciones de los niveles de iluminación (13) y monitorearon la temperatura y la humedad relativa del aire, para ello fue necesario inspeccionar el edificio y conocer su estado de conservación y sus programas de mantenimiento.

La revisión minuciosa de los objetos asociada al estudio de las condiciones ambientales, permitió comprender su estado y determinar los deterioros más frecuentes y sus posibles causas, lo que sirvió de base para proponer programas y medidas de conservación.

Las prácticas permitieron llevar a cabo algunas acciones de conservación como la actualización y elaboración de inventarios en la totalidad de las instituciones mencionadas. La realización de limpiezas y tratamientos directos sobre las obras fue preponderante en el Museo del Carmen de Villa de Leyva debido al descuido en que se encontraba la colección (fig. 7). 
En otras experiencias (Museo de Arte del Tolima), el trabajo práctico con estudiantes atendió más a las condiciones ambientales y de exhibición, especialmente en lo relacionado con el mejoramiento de los niveles de iluminación (fig. 8).

En otros casos como el de los Museos Histórico de Bolívar y de Arte Contemporáneo de la Quinta de San Pedro Alejandrino, las acciones desarrolladas se centraron más en la readecuación de las reservas aprovechando los recursos disponibles (fig. 9).

Estos trabajos han sido documentados en informes escritos y enviados a las instituciones, destacando la posibilidad de realizar acciones de conservación con pocos recursos materiales y en muy poco tiempo.

Como resultado de la aplicación de este enfoque práctico de la cátedra, se ha consolidado el área de conservación preventiva dentro de la facultad, que tiene una participación importante en proyectos de conservación a nivel nacional.

Entre estos proyectos se destaca el trabajo realizado en el Museo Antón García Bonilla ubicado en Ocaña Norte de Santander, en convenio con el Ministerio de Cultura. El proyecto comprendió el estudio y diagnóstico de las colecciones que incluían diversos tipos de objetos arqueológicos, históricos y artísticos, la realización del inventario, y acciones de conservación consistentes en limpieza y desinfección (fig. 10) debido al avanzado ataque biológico que sufrían los bienes por parte de hongos e insectos. Lo más relevante fue hacer el montaje de la exhibición y al finalizar el trabajo reabrir el museo al publico luego de 10 años de haber estado cerrado. La apertura del museo, tuvo una gran acogida por parte de la comunidad ocañera.

Otro proyecto que ha tenido un gran impacto social es el de la conservación de las momias de San Bernardo, Cundinamarca, en convenio con la Alcaldía de San Bernardo. Este Museo está ubicado en el cementerio del pueblo y exhibe un grupo de momias que se han producido de forma natural a lo largo de los últimos 50 años sin que se conozcan hasta el momento las causas que permiten este fenómeno. El trabajo comprendió el diagnóstico y acciones de conservación de las momias y 
concluyó con un taller en el teatro municipal de San Bernardo con la participación de las autoridades políticas y religiosas y gran asistencia de la comunidad.

Estos dos proyectos involucraron de manera activa a la sociedad, factor decisivo en la conservación del patrimonio cultural de una región.

Tal vez el proyecto más importante desde el punto de vista académico, es la investigación que acaba de concluir en convenio con el Ministerio de Cultura: Modelo de Conservación Preventiva para museos. El trabajo consiste en la formulación de un modelo de metodología para poder evaluar las necesidades de conservación en los museos. Se propone una herramienta sencilla, de fácil aplicación que permite una mirada integral a todos los elementos que pueden poner en riesgo la supervivencia de las colecciones y que brinda las pautas para la toma de medidas correctivas, ya sean de orden administrativo, de infraestructura y ambiente, del manejo técnico de las colecciones o partir de la relación de los museos con la sociedad. El modelo fue probado exitosamente en tres museos regionales; El Museo Casa de la Cultura Horacio Rodríguez Plata del Socorro Santander y en los museos Histórico de Mompox y Cultural de Arte Religioso de Mompox, Bolívar.

En los últimos años se ha hecho cada vez más evidente la necesidad de involucrar a toda la sociedad en la protección del patrimonio cultural, consciente de ello, la cátedra de conservación preventiva viene realizando una serie de cursos de extensión para funcionarios que de una u otra forma tienen relación con el cuidado de las colecciones patrimoniales; museólogos, arqueólogos, antropólogos, historiadores, bibliotecólogos, archivistas, arquitectos y otros profesionales afines. A estos eventos han asistido funcionarios de varias regiones del país y de países vecinos como Venezuela y Ecuador. Estos cursos han sido impartidos además fuera de la frontera de Colombia, en el año 2002 en Ciudad Bolívar (Venezuela) (14) y en el 2005 en San Salvador (El Salvador). (15)

\section{Consideraciones finales}

El desarrollo de la disciplina de la restauración en Colombia desde su aparición hace treinta años y particularmente el fortalecimiento profesional que ha tenido en la 
última década con la apertura del programa universitario, ha contado con las ciencias naturales como un eje central.

En ese sentido, las ciencias exactas al participar de manera activa en los procesos de formación de conservadores - restauradores han brindado una importante contribución en la creación de un lenguaje común que ha derivado en la adopción de esquemas interdisciplinarios por parte de los profesionales de la restauración.

Dentro de ésta dinámica, el apoyo del Laboratorio de Ciencias Naturales y la apertura de nuevos espacios laborales por parte de los restauradores, han sido factores convergentes para que otros profesionales que trabajan con el patrimonio y que tradicionalmente se habían mantenido aislados, entren a formar parte de este esquema interdisciplinar; de ese modo, tanto científicos como restauradores han comenzado a ser vistos como una necesidad en los proyectos e investigaciones que se llevan a cabo.

A pesar de las limitaciones y debilidades que existen en relación al tema del estudio del patrimonio y especialmente en relación con el acceso a nuevas tecnologías de análisis científico, el trabajo del Laboratorio ha hecho posible centralizar años de esfuerzo en una base de datos que recopila un vasto conocimiento del patrimonio colombiano y que cada día se amplía ya no únicamente con la solicitud de análisis por parte de los restauradores sino de otros públicos más amplios como arquitectos, historiadores del arte y comerciantes.

Un factor decisivo en la evolución de este proceso ha sido el apoyo del Estado, que por su relación estrecha con el programa de estudios, ha podido acompañar los avances de la academia, formulando políticas que estimulan e incluso obligan, a la ejecución de diagnósticos y estudios previos que justifiquen y sustenten los trabajos que se lleven a cabo sobre los bienes culturales muebles e inmuebles. Adicionalmente el Ministerio de Cultura ha comenzado a orientar su enfoque y sus esfuerzos, a estimular la conservación y el mantenimiento de las colecciones. 
Este complejo marco, ha brindado las condiciones para que la conservación preventiva se consolide más que como un área de conocimiento, como una filosofía de trabajo.

De igual forma la experiencia de la cátedra de conservación preventiva y los ejercicios prácticos que ha realizado, han permitido formular nuevos cuestionamientos en torno a la necesidad de involucrar a la comunidad como un agente activo y además decisivo en la ejecución de proyectos de conservación.

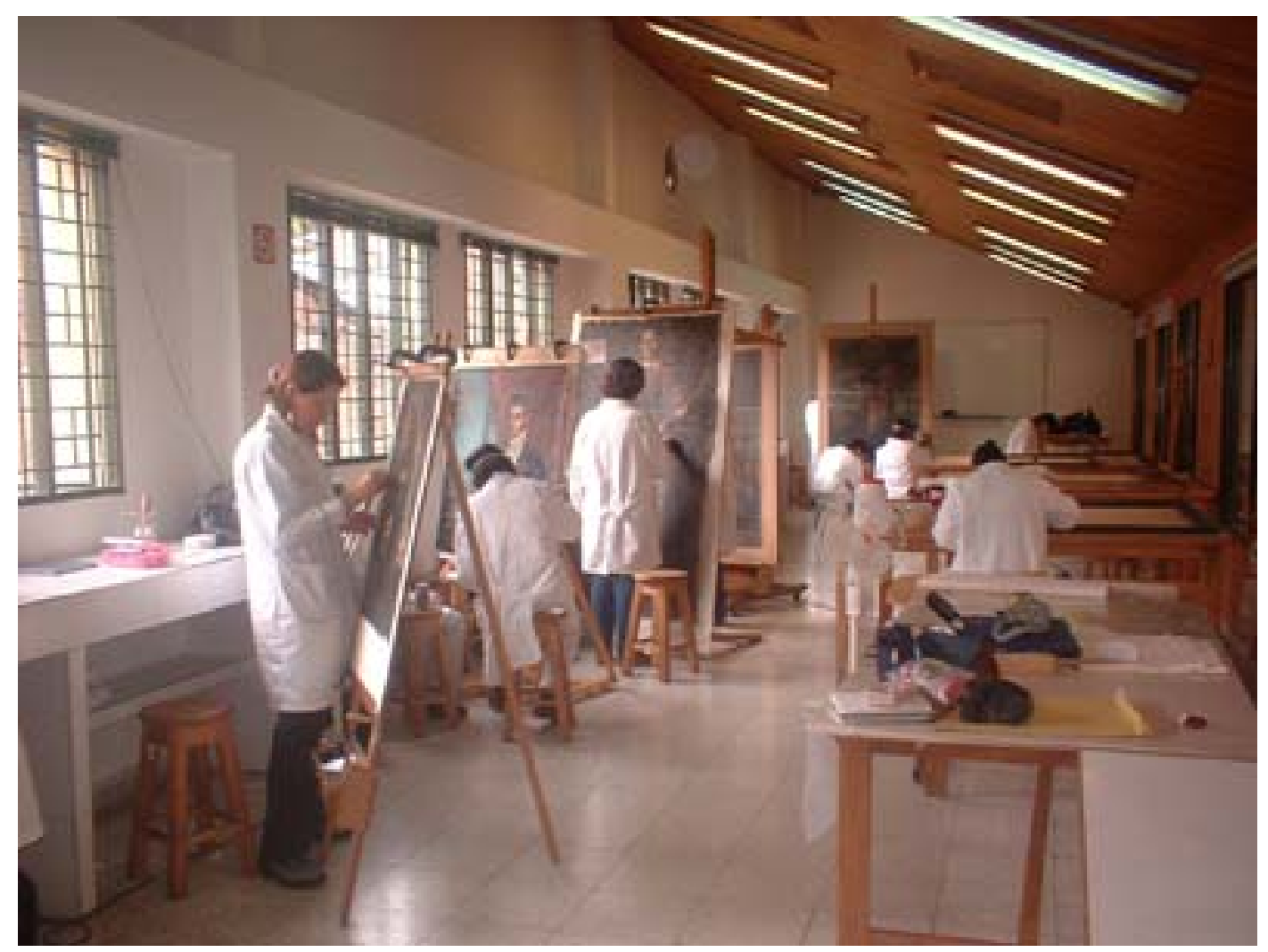

Figura 1 


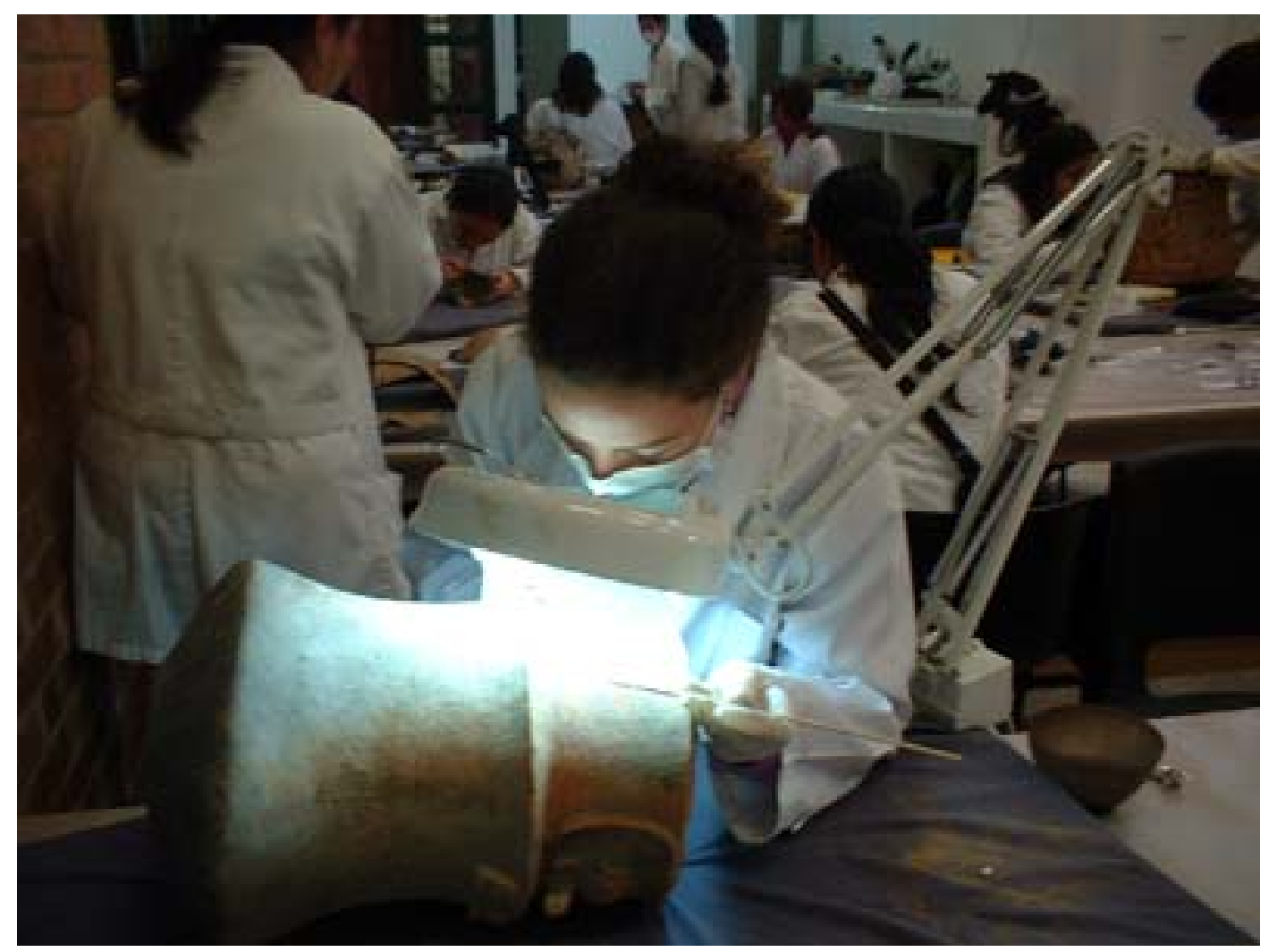

Figura 2

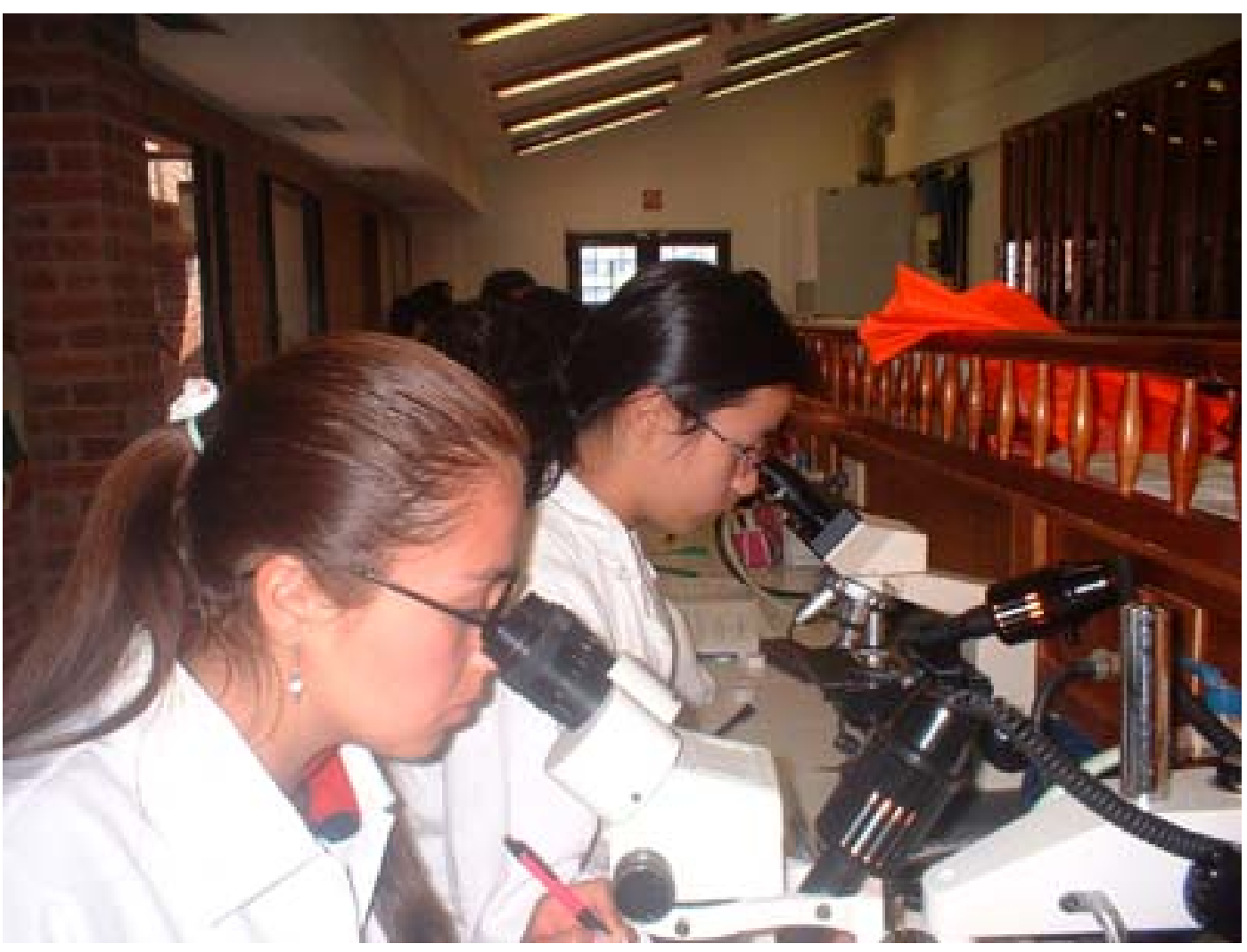

Figura 3 


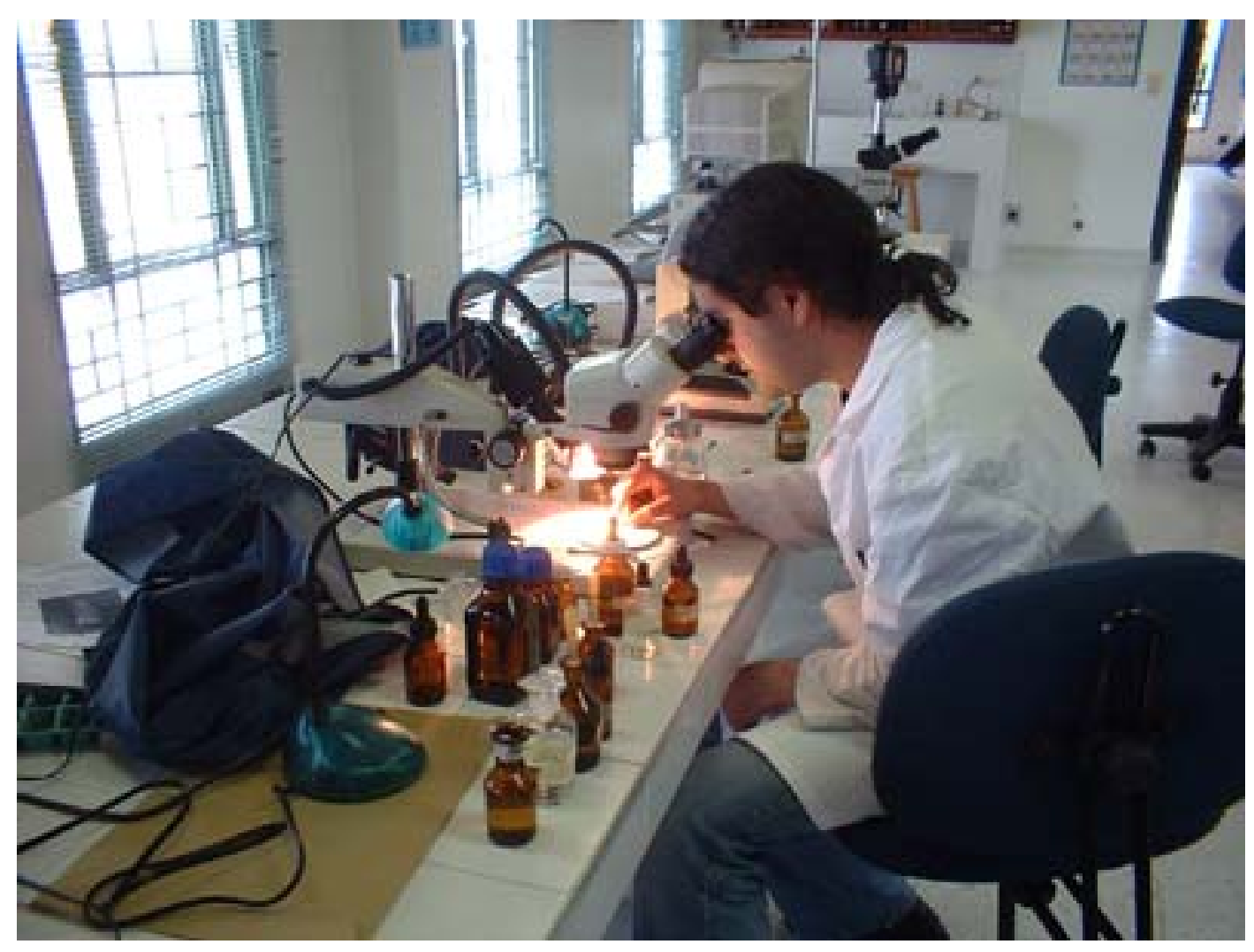

Figura 4

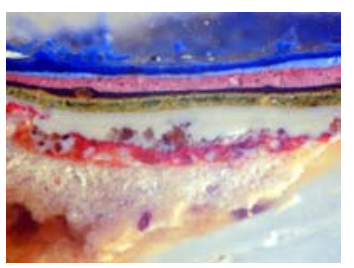

Figura 5

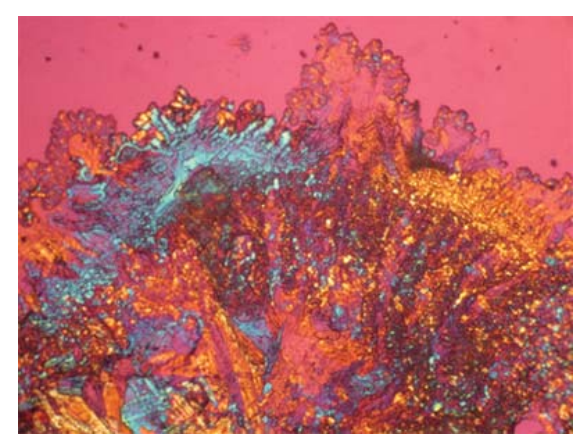

Figura 6 


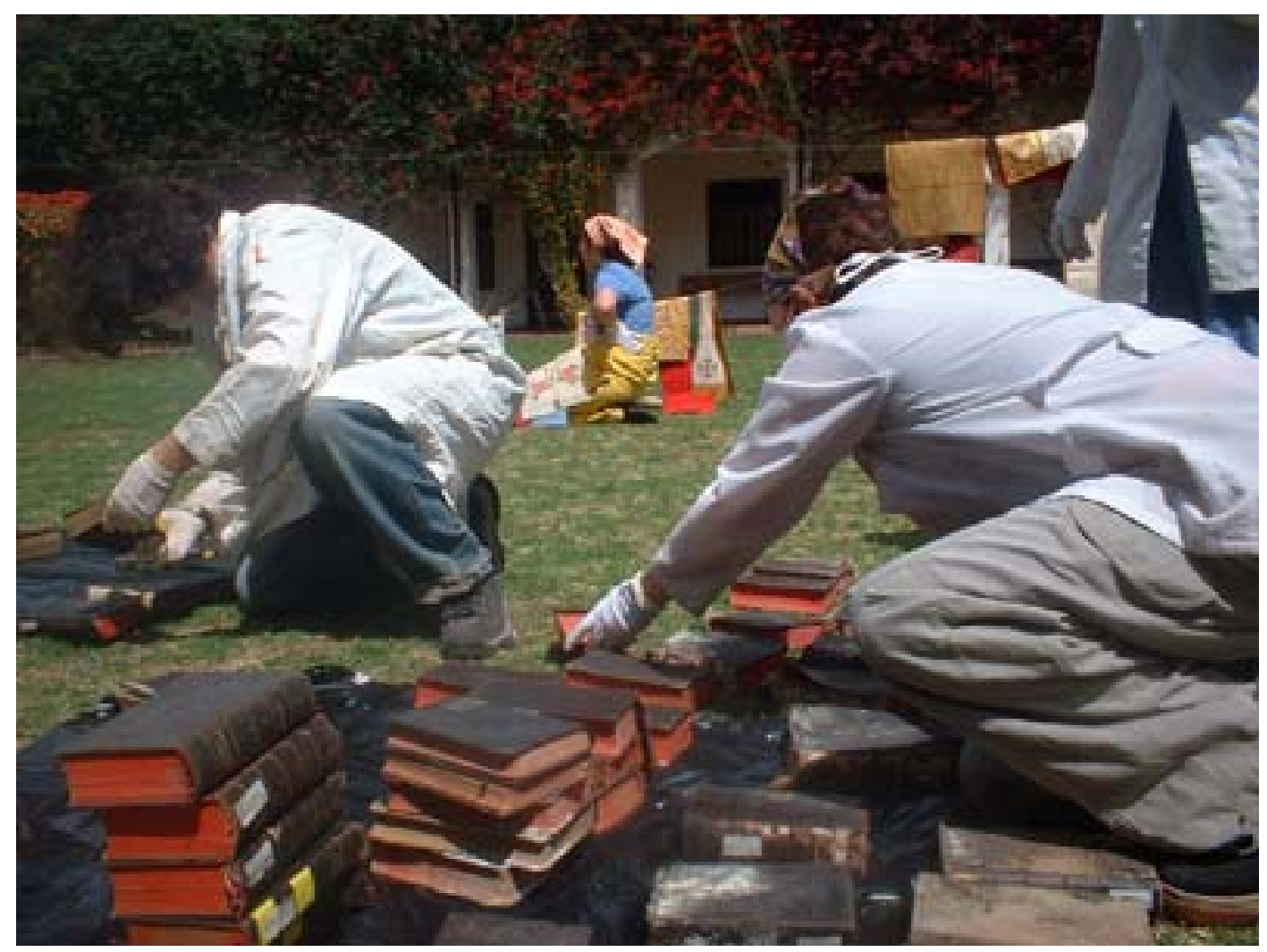

Figura 7

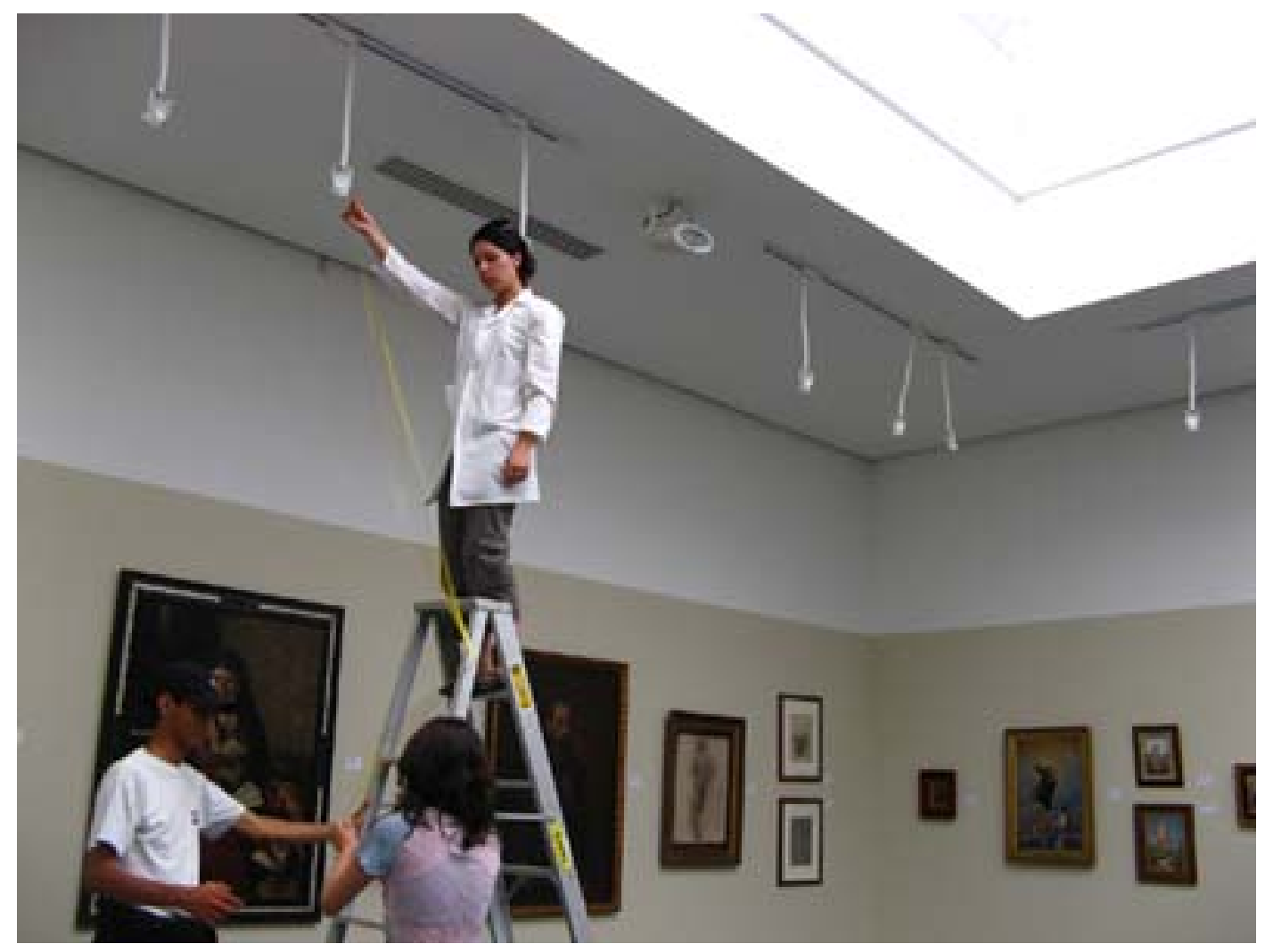

Figura 8 


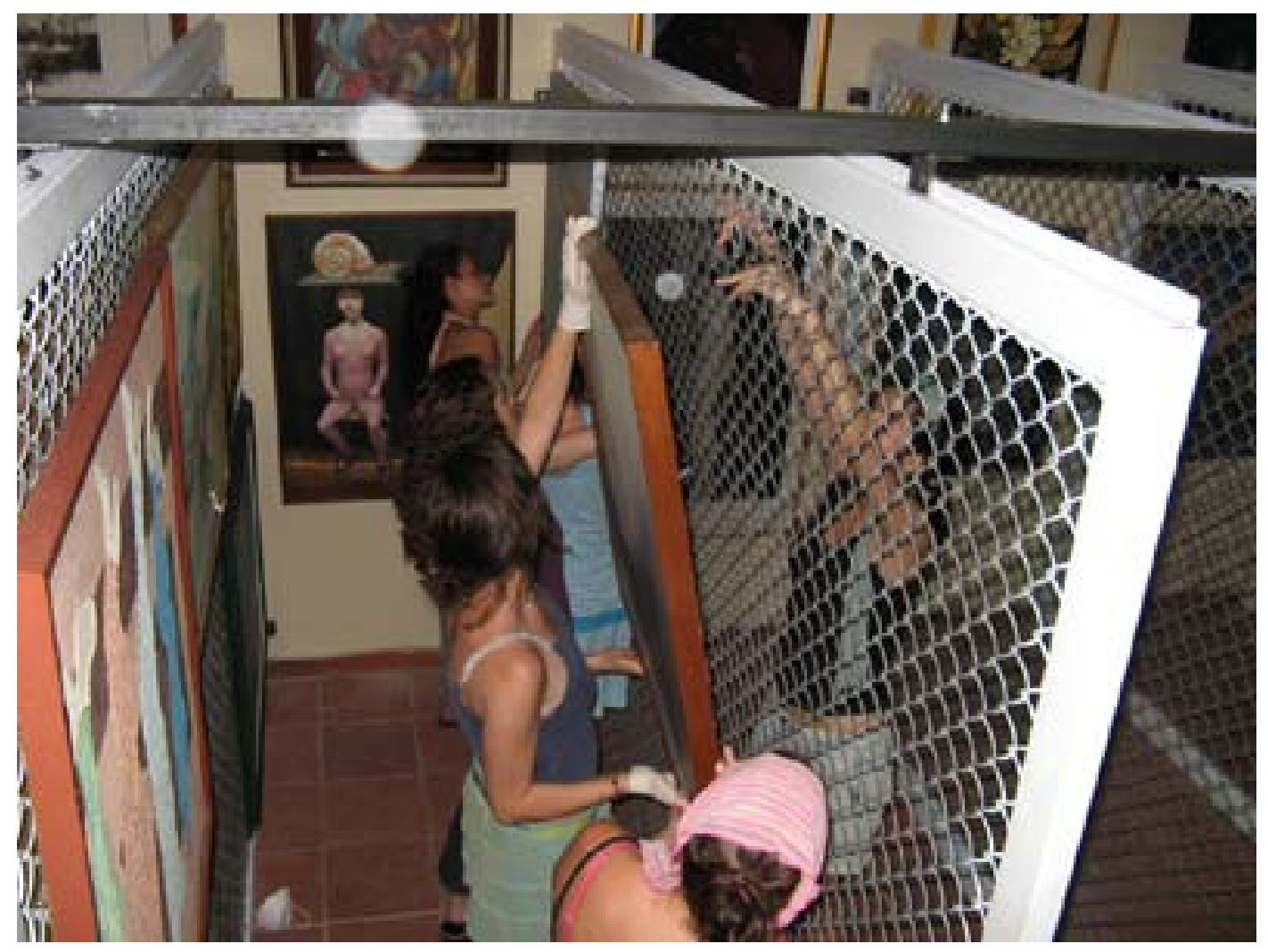

Figura 9

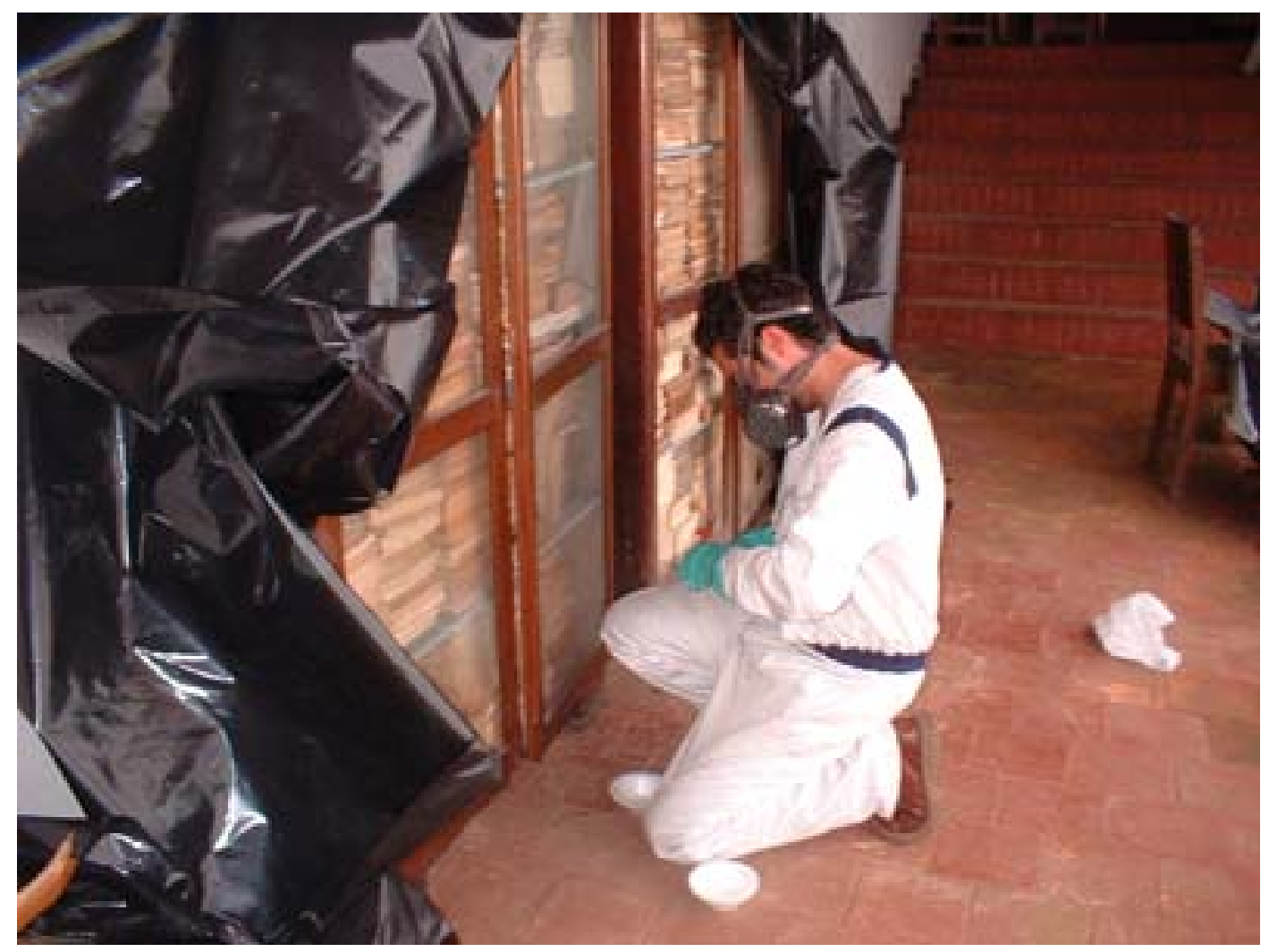

Figura 10 


\section{Notas}

(1) El Instituto Colombiano de Cultura COLCULTURA, fue el órgano del Estado encargado de dirigir las políticas culturales de Colombia y ejecutar los proyectos y recursos hasta 1991, cuando a raíz de la reforma a la constitución política, se crea el Ministerio de Cultura que asume las funciones del Instituto.

(2) Escuela de Conservación, Restauración y Museología "Manuel del Castillo Negrete" ENCRYM, adscrita al Instituto Nacional de Antropología e Historia (INAH) de México. El programa de formación profesional del ENCRYM ha tenido estrechos vínculos con el de Colombia y la colaboración entre ambas entidades ha permitido el intercambio de docentes y la publicación de trabajos.

(3) Actualmente el área de microbiología del Laboratorio acaba de terminar una investigación que contó con la participación de restauradores, encaminada a probar productos biocidas para bienes documentales, extraídos de productos naturales como la canela y el clavo de fácil acceso y bajo costo en Colombia.

(4) Análisis de morteros y decoraciones murales. Lovendegem. Instituto VandemBorre - Lauwers, Bélgica. Informe técnico. Laboratorio de Ciencias Naturales. Universidad Externado de Colombia, 2007.

(5) Análisis de la pintura mural de la Iglesia de Cuarahuara de Carangas. Edgar Ramiro Mendieta. Oruro, Bolivia. Informe técnico. Laboratorio de Ciencias Naturales. Universidad Externado de Colombia, 2001.

(6) Análisis de pigmentos de dos obras del pintor Juan Francisco González. Centro de Conservación, Restauración y Estudios Artísicos CREA. Santiago de Chile, Chile. Informe técnico. Laboratorio de Ciencias Naturales. Universidad Externado de Colombia, 2004.

(7) Estudio de materiales y estratigrafías de la fachada del Templo de Santo Domingo de Guzmán. Haydeé Orea Magaña. San Cristóbal de las Casa, Chiapas, México. Informe técnico. Laboratorio de Ciencias Naturales. Universidad Externado de Colombia, 2006.

(8) Análisis de materiales. Muro arqueológico de las ruinas de la antigua ciudad de Panamá. Maria de la Paz Gómez. Ciudad de Panamá, Panamá. Informe técnico. Laboratorio de Ciencias Naturales. Universidad Externado de Colombia, 2007.

(9) La base de datos del centro de documentación del Laboratorio cuenta con casi mil quinientos informes y un banco con más de doce mil muestras.

(10) MATIZ, Paula; OVALLE, Angela. Conservación preventiva en museos: evaluación de riesgos. Bogotá: Universidad Externado de Colombia, 2006.

(11) Los materiales producidos por las experiencias del Consorcio están disponibles en la página web: http://www.uexternado.edu.co/conservacionpreventiva

(12) Se probaron las metodologías del Instituto Canadiense de Conservación (CCI - ICC) y del Instituto Getty de Conservación (GCl). 
(13) Para la medición de los niveles de iluminación se empleó en las prácticas una metodología propia publicada por el ICOM-CC en la 14 reunión trienal del 12 - 16 de septiembre. La Haya, 2005.

(14) Curso de conservación preventiva para colecciones de bienes culturales. IPH. Ciudad Bolívar, Venezuela. 2002.

(15) Curso de conservación preventiva para arquitectos. CONCULTURA. San Salvador, El Salvador. 2005

\section{Referencias Bibliográficas}

BERGEON, Segolene. La interdisciplinariedad en la conservación - restauración de los bienes culturales. Restauración Hoy, Bogotá D.C., n. 8, p. 14-26, 1995.

CADAVID, Lucia. Origen, significado y desarrollo de la restauración en Colombia. Restauración Hoy, Bogotá D.C., n. 6, p. 30-41, 1994.

EU-ARTECH. Conclusions of the internacional meeting science and technology on Cultural Heritage. EUARTECH, 2007. Disponible en: http://www.eu-artech.org/files/Final-conclusions-last.pdf. Consultado el 25 jun. 2007.

FERNANDEZ, Mario Omar. El estudio de la iluminación en los museos una metodología al alcance de todos. En: ICOM - CC TRIENNIAL MEETING, 14., 2005, The Hague. Preprints... The Hague: ICOM Committee for Conservation, 2005. p. 689-692. v. 2.

GARCÉS, Jhon. Evaluación de varios ingredientes activos con actividad biocida, sobre probetas de cuero y pergamino utilizadas para la encuadernación del patrimonio mueble colombiano. En: TALLER SOBRE LA CONSERVACIÓN DEL PATRIMONIO DOCUMENTAL Y LA PREVENCIÓN CONTRA CATÁSTROFES EN PAÍSES DE CLIMA TROPICAL, 2007, La Habana (Cuba). La Habana: Archivo Nacional de la República de Cuba, [2007].

GETTY CONSERVATION INSTITUTE. The Conservation Assessment: a proposed model for evaluating museum environmental management needs. Los Angeles (USA): GCI, 1999.

HERRAEZ, Juan; LORITE, Miguel A. La conservación preventiva de obras de arte. Grupo Español IIC, 1999. Disponible en: http://ge-iic.com/gruposconservacionpre/CONSERVACIONPREVENTIVA.pdf. Consultado el 26 jun. 2007.

LEMP, Cecilia M. Posicionamiento de la conservación - restauración en Chile como disciplina universitaria. Conserva (Revista del Centro Nacional de conservación y Restauración DIBAM), Santiago de Chile, n. 8, p. 3155, 2004.

MATIZ, Paula; OVALLE, Angela. Conservación preventiva en museos: evaluación de riesgos. Bogotá: Universidad Externado de Colombia, 2006. 
MICHALSKI, Stefan. Conservación de las colecciones de museos. Enfoque sistemático. Canadá: Canadian Conservation Institute (CCI - ICC), 1992.

VARAS, Ignacio. Conservación de bienes culturales: teoría, historia, principios y normas. Madrid: Cátedra, 1999. 90

* Mario Omar Fernández R. MsC. Ingeniero Químico. Instituto Mendeleiev. Moscú. Director del Laboratorio de Ciencias Naturales Facultad de Estudios del Patrimonio Cultural Universidad Externado de Colombia. marioo.fernandez@uexternado.edu.co

** David Cohen D. Restaurador de Bienes Muebles. Universidad Externado de Colombia Director del programa de Conservación y Restauración de Bienes Muebles Facultad de Estudios del Patrimonio Cultural Universidad Externado de Colombia. david.cohend@uexternado.edu.co 